
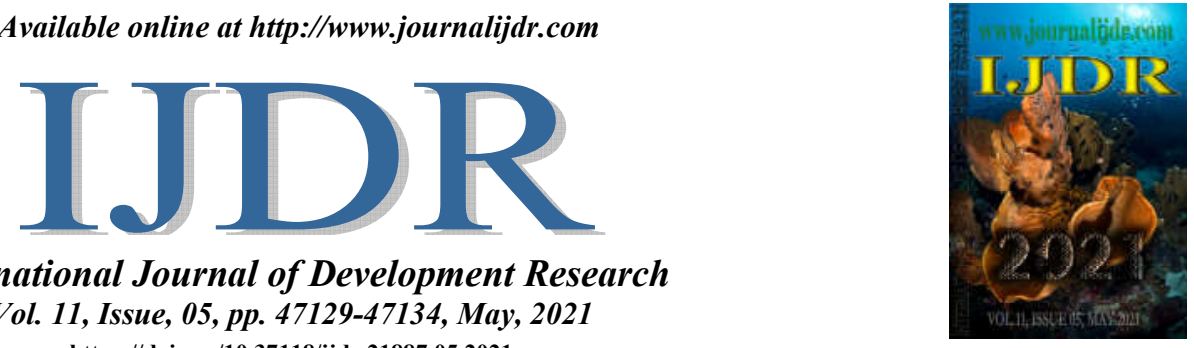

ISSN: 2230-9926

International Journal of Development Research

Vol. 11, Issue, 05, pp. 47129-47134, May, 2021

https://doi.org/10.37118/ijdr.21887.05.2021

OPEN ACCESS

RESEARCH ARTICLE

\title{
GESTÃO DE RESÍDUOS SÓLIDOS NOS SERVIÇOS DE SAÚDE: UMA REVISÃO INTEGRATIVA DA LITERATURA
}

\section{${ }^{*}$ Andressa Pedroza Pereira and Maria de Fátima Martins}

\author{
Programa de Pós-Graduação em Engenharia e Gestão de Recursos Naturais, Universidade Federal de Campina \\ Grande, Campina Grande-PB
}

\section{ARTICLE INFO}

\section{Article History:}

Received $19^{\text {th }}$ February, 2021

Received in revised form

$23^{\text {rd }}$ March, 2021

Accepted $14^{\text {th }}$ April, 2021

Published online $30^{\text {th }}$ May, 2021

\section{Key Words:}

Resíduos sólidos, Serviços de saúde, gestão de resíduos, Plano de

Gerenciamento de Resíduos de Serviços de Saúde

*Corresponding autho: Andressa Pedroza Pereira

\begin{abstract}
Certos tipos de resíduos merecem destaque dentro do contexto dos resíduos sólidos, são aqueles provenientes dos serviços de saúde, os chamados Resíduos de Serviços de Saúde (RSS). Estes resíduos merecem uma atenção maior devido às suas características físicas, químicas e biológicas, configurando-se em um aglomerado potencial de microrganismos. O gerenciamento impróprio dos RSS expõe a sig $\neg$ nificantes riscos os pacientes, os trabalhadores da saúde, os manipuladores destes resíduos, a comunidade em geral e o meio ambiente como um todo. O estudo tem como objetivo identificar o que a literatura evidencia sobre a gestão dos RSS. Foiutilizado como metodologia a revisão integrativa da literatura, tendo como base de dados a Scielo e a Web of Science, sendo selecionados 23 artigos, mediante critérios de inclusão/ exclusão. Os resultados demonstraram que a maior parte dos estabelecimentos de saúde estudados não tinham uma gestão de RSS adequada, e a maioria dos profissionais/acadêmicos também não possuíam conhecimento suficiente acerca da temática. A gestão dos RSS necessita de ajustes em relação à construção de Planos de Gerenciamento de Resíduos de Saúde, com a implementação de todas as suas etapas e ênfase nas capacitações dos profissionais que lidam diretamente com estes resíduos.
\end{abstract}

Copyright (C) 2021, Andressa Pedroza Pereira Maria de Fátima Martins This is an open access article distributed under the Creative Commons Attribution License, which permits unrestricted use, distribution, and reproduction in any medium, provided the original work is properly cited.

Citation: Andressa Pedroza Pereira Maria de Fátima Martins, 2021. "Gestão de resíduos sólidos nos serviços de saúde: uma revisão integrativa da literature", International Journal of Development Research, 11, (05), 47129-47134.

\section{INTRODUCTION}

A partir do século XVIII, o advento da Revolução Industrial trouxe consigo mudanças globais, entre elas, a mudança dos meios de trabalho que deixaram de ser exclusivos do campo e passaram a ocupar os centros das cidades. A intensa urbanização atrelada ao crescimento populacional exponencial e ao consumismo elevado, resultou na problemática da inadequada gestão dos resíduos sólidos e nos consequentes impactos ambientais e sociais decorrentes desta situação $^{(1)}$. Segundo o Panorama dos Resíduos Sólidos no Brasil de 2017, a região Nordeste apresenta o menor índice de cobertura de coleta de Resíduos Sólidos Urbanos (RSU), sendo a única abaixo dos $80 \%$, apesar de responder por $25 \%$ dos resíduos gerados no país. Ademais, a destinação adequada dos RSU coletados pelos municípios avançou minimamente, enquanto o volume enviado para lixões apresentou um crescimento de 3\% de 2016 para 2017, com 1.610 cidades fazendo uso dessas unidades, as quais são a pior forma de destinação dos materiais descartados, pois não apresentam nenhuma proteção ambiental e causam severos impactos na saúde das pessoas $^{(2)}$. Um tipo de resíduos que merecem destaque dentro deste contexto, são aqueles provenientes dos serviços de saúde - os Resíduos de Serviços de Saúde (RSS), mais conhecidos como "lixo odontológicas, veterinárias, farmácias, centros de pesquisa farmacologia e saúde, medicamentos vencidos, necrotérios, funerárias, medicina legal e barreiras sanitárias ${ }^{(3)}$. Em destaque, quanto à legislação concernente aos resíduos de serviços de saúde (RSSs), é possível destacar as Resoluções da Diretoria Colegiada (RDC) da ANVISA, sendo elas a RDC n 306/2004, compatível com a Resolução n ${ }^{\circ} 358 / 2005$, do Conselho Nacional do Meio Ambiente (CONAMA), que após o advento da Política Nacional de Resíduos Sólidos (PNRS), instituída pela Lei 12.305/2010 necessitou de revisões para que se tornasse compatível com a realidade e evoluções da época, dando espaço a RDC $n^{\circ} 222 / 2018^{(4,5)}$. Embora componham uma pequena parcela do montante de resíduos gerados pelos municípios, os RSS merecem uma atenção maior devido às suas características físicas, químicas e biológicas, sendo um aglomerado potencial de microrganismos. Decorrente dessa gama de características, com diferentes propriedades, estes recursos requerem distintos e específicos métodos de gestão para seu tratamento e/ou disposição final, segundo sua classificação (MELO et al, 2015). O gerenciamento impróprio destes resíduos expõe a significantes riscos pacientes, trabalhadores da saúde, manipuladores dos resíduos, a comunidade em geral e o meio ambiente como um todo ${ }^{(6)}$. Refletindo sobre a problemática dos RSS, infere-se que o gerenciamento adequado destes resíduos é imprescindível para a saúde pública e uma responsabilidade de todos; e o seu descarte inadequado tem 
produzido danos à saúde do trabalhador, da comunidade e ao ambiente capazes de colocar em risco e comprometer os recursos naturais e a qualidade de vida das atuais e futuras gerações. Tendo em vista a necessidade de um gerenciamento de resíduos eficiente nos serviços de saúde, com ênfase na sustentabilidade em seus processos assistenciais, a presente pesquisa traz a seguinte questão norteadora: o que a literatura evidencia sobre a gestão dos resíduos sólidos nos serviços de saúde? Neste sentido, o presente estudo tem como objetivo identificar o que a literatura evidencia sobre a gestão dos resíduos sólidos nos serviços de saúde.

\section{MATERIAIS E MÉTODOS}

A presente pesquisa trata-se de uma revisão integrativa, um tipo de Prática Baseada em Evidências (PBE), que tem como finalidade analisar o conhecimento construído em estudos precedentes sobre uma determinada temática, incentivando os profissionais da área da saúde a atuarem em busca de estudos $\operatorname{clínicos}^{(7)}$. Ao longo do tempo, com o crescimento e complexidade de conhecimentos na área da saúde, requereu-se a construção de artigos fundamentados cientificamente, que apresentem etapas metodológicas delimitadas e concisas, viabilizando ao profissional de saúde o melhor uso das evidências identificadas em inúmeras pesquisas ${ }^{(8)}$.

A elaboração dessa revisão integrativa ocorreu em seis etapas distintas descritas a seguir: Primeira etapa - Identificação do tema e seleção da hipótese ou questão de pesquisa para a elaboração da revisão integrativa. A revisão teve início com a definição de um problema e formulação de uma questão de pesquisa, neste caso, com relevância para área de saúde. Assim, a questão norteadora da presente pesquisa é: o que a literatura evidencia sobre a gestão dos resíduos sólidos nos serviços de saúde?

Segunda etapa - Estabelecimento de critérios para inclusão e exclusão de estudos/amostragem ou busca na literature

Para a busca dos estudos foram utilizadas as bases de dados: ScientificElectronic Library Online (SCIELO) e Web of Science. Utilizou-se como estratégia de pesquisa os seguintes descritores em saúde: resíduos sólidos, serviços de saúde e gestão de resíduos. Os critérios utilizados para a seleção foram: todos os artigos disponíveis nas bases de dados supracitadas, durante todos os anos disponíveis (2005 a 2020); nos idiomas português, inglês e espanhol; disponíveis online na íntegra; que apresentem discussão relevantes sobre a gestão dos resíduos sólidos nos serviços de saúde e indexados nas bases de dados da SciELO e Web of Science. Os critérios de exclusão foram artigos que se apresentassem repetidos em mais de uma base de dados e que não contemplassem os objetivos da investigação. Inicialmente foi realizado dois entrecruzamentos de dois descritores distintos na primeira base de dados (Scielo), na qual, no primeiro cruzamento (resíduos sólidos, serviços de saúde) foram encontrados 35 artigos, que após aplicação dos critérios de exclusão perfez 07 artigos e no segundo cruzamento (gestão de resíduos; serviços de saúde) foram 19 artigos, e utilizados 09 após os critérios de exclusão. Na segunda base de dados (Web of Science), foram utilizados os mesmos entrecruzamentos da primeira base (Scielo), porém no primeiro cruzamento (resíduos sólidos, serviços de saúde), não foram encontrados artigos que pudessem responder ao objetivo do estudo, configurando-se como uma das limitações da pesquisa. Logo, no segundo cruzamento (gestão de resíduos, serviços de saúde) foram encontrados 32 artigos, 21 após os critérios de inclusão e utilizados 07, após os critérios de exclusão. A fim de alcançar o objetivo proposto, nesta base de dados foram utilizadas categorias e áreas de pesquisa contidas na própria base, a saber: categorias - serviços de política de saúde, saúde ocupacional pública ambiental, ciências multidisciplinares, farmacologia, medicina geral interna, enfermagem, medicina tropical, administração pública, recursos hídricos e tecnologia da ciência verde sustentável; áreas de pesquisa saúde ocupacional pública ambiental, serviços de ciências da saúde, medicina interna geral, tecnologia da ciência e outros tópicos, farmacologia, administração pública, medicina tropical, recursos hídricos e enfermagem. Ao observar o quadro abaixo, foram encontrados 54 artigos e utilizados apenas 23. Esse dado reflete a fragilidade quanto à produção científica abordando a gestão dos resíduos sólidos nos serviços de saúde. É necessário investir mais na área e sensibilizar os profissionais para realizarem pesquisas que abordem o tema com o objetivo de melhorar a gestão conforme a legislação vigente e aumentar o referencial teórico nas bases de dados.

Quadro 1. Quantidade de artigos encontrados

\begin{tabular}{|c|c|c|c|c|}
\hline CRUZAMENTO & \multicolumn{2}{|c|}{ SCIELO } & \multicolumn{2}{|c|}{ WEB OF SCIENCE } \\
\hline Resíduos Sólidos X & Encontrado & Utilizado & Encontrado & Utilizado \\
\hline Serviços de Saúde & 35 & 07 & . & - \\
\hline $\begin{array}{l}\text { Gestão de Resíduos } \\
\text { X Serviços de } \\
\text { Saúde }\end{array}$ & 19 & 09 & 32 & 07 \\
\hline $\begin{array}{l}\text { TOTAL } \\
\text { ENCONTRADOS }\end{array}$ & \multicolumn{2}{|c|}{54} & \multicolumn{2}{|c|}{32} \\
\hline $\begin{array}{l}\text { TOTAL } \\
\text { UTILIZADOS }\end{array}$ & \multicolumn{2}{|c|}{16} & \multicolumn{2}{|c|}{07} \\
\hline
\end{tabular}

Fonte: AUTORES, 2020

Terceira etapa - Definição das informações a serem extraídas dos estudos selecionados/categorização dos estudos: Para categorizar os artigos selecionados e definir os dados a serem extraídos dos estudos foi construído uma tabela onde constam informações importantes como procedência, título, nomes dos autores, periódico (vol, $\mathrm{n}^{\mathrm{o}}$, pág, ano), considerações/temática.

Quarta etapa - Avaliação dos estudos incluídos na revisão : O delineamento desta etapa deu-se através de leituras exploratórias que avaliaram as pesquisas selecionadas para a revisão de forma crítica, seletiva e analítica, interpretando os resultados discutidos nas pesquisas.

Quinta etapa - Interpretação dos resultados: Esta etapa consistiu na análise das informações coletadas, a qual requereu interpretação e síntese dos resultados. Por meio desta etapa, pode-se construir comparações entre as informações coletadas e o referencial teórico, o que permite identificar lacunas e projetar novas pesquisas ${ }^{(9)}$.

Sexta etapa - Publicação e comunicação dos achados : A última etapa retrata a apresentação da síntese do conhecimento produzido, ou seja, consiste em apresentar de forma criteriosa uma revisão com todo o trajeto percorrido pelo pesquisador, evidenciando de forma clara e completa os resultados, permitindo assim que o leitor avalie de forma crítica e criteriosa os resultados.

\section{RESULTADOS}

Dos 23 artigos escolhidos identificou-se que a maioria é produção de periódicos nacionais, na área da saúde, onde estes representaram $60,8 \%(n=14)$ dos estudos analisados, conforme os dados descritos na tabela I. Do total de artigos, apenas 01 se configurou como um estudo internacional, ou seja $4,3 \%$ dos achados. Ainda conforme os dados da Tabela 1, a maioria dos artigos foram encontrados no idioma português $(65,2 \%)$, o que confirma a maior parte da produção na temática analisada ser nacional.

Tabela 1. Áreas de Pesquisa dos Periódicos, Países e Idiomas dos

\begin{tabular}{lcc}
\hline Áreas de Pesquisa dos Periódicos & $\mathrm{N}$ & $\%$ \\
\hline Saúde & 14 & $60,8 \%$ \\
Engenharia Sanitária e Ambiental & 5 & $21,7 \%$ \\
Gestão & 3 & $13 \%$ \\
Ciências & 1 & $4,3 \%$ \\
TOTAL & 23 & $100 \%$ \\
País & $\mathrm{N}$ & $\%$ \\
Brasil & 22 & $95,6 \%$ \\
Nigéria & 1 & $4,3 \%$ \\
TOTAL & 23 & $100 \%$ \\
Idioma & $\mathrm{N}$ & $\%$ \\
Português & 15 & $65,2 \%$ \\
Inglês & 3 & $13 \%$ \\
Português/Inglês & 5 & $21,7 \%$ \\
TOTAL & 23 & $100 \%$ \\
\hline
\end{tabular}

Fonte: AUTORES, 2020 
Tabela 2. Distribuição das pesquisas incluídas na revisão integrativa de acordo com procedência, título, nomes dos autores, periódico (vol, $\mathbf{n}^{\circ}$, pág, ano), considerações/temática, referentes aos anos de 2013-2020

\begin{tabular}{|c|c|c|c|c|}
\hline Procedência & Título & Autores & $\begin{array}{l}\text { Periódico (vol, } \mathrm{n}^{\mathrm{o}} \text {, } \\
\text { pág, ano) }\end{array}$ & Considerações/temática \\
\hline Scielo & $\begin{array}{l}\text { Desafios na gestão de resíduos } \\
\text { de estabelecimentos de saúde } \\
\text { públicos perante a RDC } \\
222 / 18^{(10)}\end{array}$ & Delevati, DS et al & $\begin{array}{l}\text { Saúde } \\
\text { debate } 43 \text { (no.spe3 } \\
\text { ):190-199, Jan } \\
2020 .\end{array}$ & $\begin{array}{l}\text { Verificaram-se desafios na gestão dos Resíduos de Serviços de } \\
\text { Saúde (RSS) relativos à ausência de plano de gerenciamento, falha } \\
\text { no manejo, falta de abrigo externo, falhas na tomada de decisão e } \\
\text { quanto à responsabilidade técnica; necessitando de maior } \\
\text { visibilidade em meio aos profissionais da saúde, especialmente no } \\
\text { campo da educação permanente. }\end{array}$ \\
\hline Scielo & $\begin{array}{l}\text { Condições da prestação dos } \\
\text { serviços ambientais de coleta e } \\
\text { destinação de resíduos de } \\
\text { serviços de saúde em unidades } \\
\text { básicas de saúde na cidade de } \\
\text { Pelotas, RS, Brasil }{ }^{(11)}\end{array}$ & $\begin{array}{l}\text { Neves BC, Lima } \\
\text { EPP }\end{array}$ & $\begin{array}{l}\text { EngSanitAmbient } \\
\text { 24(1): } 61-69 \\
\text { jan/fev } 2019 \text {. }\end{array}$ & $\begin{array}{l}\text { Identificou que as condições de armazenamento temporário interno } \\
\text { e externo nem sempre estão de acordo com o que recomenda a RDC } \\
\mathrm{n}^{\mathrm{o}} 306 / 04 \text {, podendo oferecer riscos ao ambiente e à população } \\
\text { exposta. Além disso, nem sempre é feito o uso de uniformes e EPIs } \\
\text { adequados pelos profissionais responsáveis pela limpeza interna nas } \\
\text { unidades. }\end{array}$ \\
\hline Web of Science & $\begin{array}{l}\text { Role of National Policy in } \\
\text { Improving Health Care Waste } \\
\text { Management in Nigeria }\end{array}$ & Ezirim I, Agbo F & $\begin{array}{l}\text { J Health Pollut } \\
8(19), \text { setembro } \\
2018 .\end{array}$ & $\begin{array}{l}\text { Constatou-se que } 89,2 \% \text { das unidades de saúde segregaram resíduos } \\
\text { no ponto de geração. } 44,8 \% \text { de todas as instalações possuíam planos } \\
\text { de gerenciamento de resíduos de saúde desenvolvidos a partir da } \\
\text { política nacional de gerenciamento de resíduos de saúde. }\end{array}$ \\
\hline Web of Science & $\begin{array}{l}\text { Diagnosisof Hospital Waste } \\
\text { Management in Vale do Rio } \\
\text { Pardo - Rio Grande do Sul, } \\
\text { Brazil }^{(13)}\end{array}$ & $\begin{array}{l}\text { Kist LT, Fernanda } \\
\text { Rosa FR, Moraes } \\
\text { JAR, Machado } \\
\text { EL }\end{array}$ & $\begin{array}{l}\text { Rev. Gest. } \\
\text { Ambient. } \\
\text { Sustentabilidade } \\
7(3): \text { 554-569, } \\
\text { set/dez } 2018 \text {. }\end{array}$ & $\begin{array}{l}\text { Observou-se que há falta de informações sobre a geração de } \\
\text { resíduos e isso contribui para um inadequado gerenciamento de } \\
\text { RSS, que pode causar riscos ao trabalhador, saúde pública e meio } \\
\text { ambiente. Ainda aponta a falta de uma política de treinamento que } \\
\text { concentra-se na questão da gestão de resíduos. }\end{array}$ \\
\hline Web of Science & $\begin{array}{l}\text { Assessment of The Waste } \\
\text { Management in Basic Health } \\
\text { Units From a South Brazilian } \\
\text { City }{ }^{(14)}\end{array}$ & $\begin{array}{l}\text { Teixeira MV, } \\
\text { Echevarría- } \\
\text { Guanilo ME, } \\
\text { Knuth FG, Ceolin } \\
\text { T }\end{array}$ & $\begin{array}{l}\text { J. res.: fundam. } \\
\text { care. Online } \\
\text { 10(3): } 824-83 \\
2018 \text {. }\end{array}$ & $\begin{array}{l}\text { Observou-se ausência de plano de gestão. As unidades apresentam } \\
\text { importantes fragilidades no processo de gerenciamento dos RSS e } \\
\text { apresentam fraqueza significativa na gestão e gestão de } \\
\text { profissionais de saúde, associados à falta de qualificação } \\
\text { profissional, bem como o desconhecimento da legislação atual que } \\
\text { lida com resíduos de cuidados de saúde. }\end{array}$ \\
\hline \multirow[t]{2}{*}{ Web of Science } & $\begin{array}{l}\text { Use of performance indicators } \\
\text { to assess the solid waste } \\
\text { management of health services } \\
(15)\end{array}$ & $\begin{array}{l}\text { Assis MC, Gomes } \\
\text { VAP, Balista WC, } \\
\text { Freitas RR }\end{array}$ & $\begin{array}{l}\text { A. Acad. Bras. Ciê } \\
\text { nc. } 89(3): 2245- \\
60, \text { outubro } 2017 \text {. }\end{array}$ & 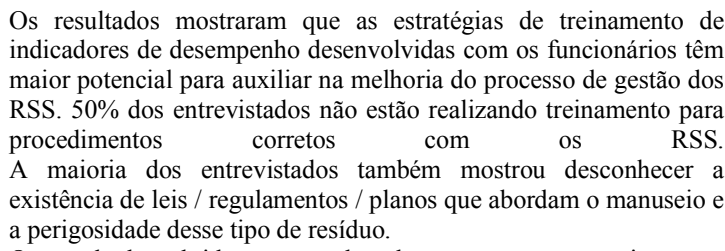 \\
\hline & $\begin{array}{l}\text { Recuperação de papéis } \\
\text { recicláveis: Aspectos da } \\
\text { reciclagem e do } \\
\text { reaproveitamento em ambiente } \\
\text { hospitalar }^{(16)}\end{array}$ & $\begin{array}{l}\text { Melo SB, Zajac } \\
\text { MAL, Régis MM, } \\
\text { Joyce Oliveira } \\
\text { JES }\end{array}$ & $\begin{array}{l}\text { Anais do VI } \\
\text { SINGEP - São } \\
\text { Paulo - SP -Brasil } \\
-13 \text { e } 14 / 11 / 2017\end{array}$ & $\begin{array}{l}\text { Os resultados obtidos no estudo demonstram que existe um } \\
\text { considerável percentual de resíduos passíveis de reciclagem ou } \\
\text { reaproveitamento sendo descartados de forma irregular em } \\
\text { recipientes do grupo A. }\end{array}$ \\
\hline Scielo & $\begin{array}{l}\text { Avaliação do gerenciamento } \\
\text { dos resíduos de medicamentos e } \\
\text { demais resíduos de serviços de } \\
\text { saúde na Região Serrana de } \\
\text { Santa Catarina }{ }^{(17)}\end{array}$ & $\begin{array}{l}\text { Amarante JAS, } \\
\text { Siegloch RTD, } \\
\text { Siegloch AE }\end{array}$ & $\begin{array}{l}\text { EngSanitAmbient } \\
\text { 22(2):317-326, } \\
\text { mar/abr } 2016\end{array}$ & $\begin{array}{l}\text { Os resultados evidenciaram deficiências no gerenciamento dos } \\
\text { resíduos de medicamentos, assim como dos demais resíduos, nas } \\
\text { etapas de segregação e armazenamento em instituições de } \\
\text { atendimento humano e veterinário, com poucas exceções. }\end{array}$ \\
\hline \multirow[t]{2}{*}{ Scielo } & $\begin{array}{l}\text { Solid waste management in } \\
\text { primary healthcare centers: } \\
\text { application of a facilitation tool } \\
\text { (18) }\end{array}$ & $\begin{array}{l}\text { Moreira AMM, } \\
\text { Günther, WMR }\end{array}$ & $\begin{array}{l}\text { Rev. Latino-Am. } \\
\text { Enfermagem 24: } \\
1-9, \text { Ago } 2016 .\end{array}$ & 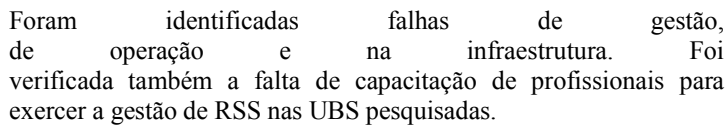 \\
\hline & $\begin{array}{l}\text { Análise da gestão e } \\
\text { gerenciamento dos resíduos de } \\
\text { serviços de saúde (RSS) do } \\
\text { Hospital de Emergência de }\end{array}$ & $\begin{array}{l}\text { Maders GR, } \\
\text { Cunha, HFA }\end{array}$ & $\begin{array}{l}\text { EngSanit } \\
\text { Ambient.20(3): } \\
379-388, \text { jul/set } \\
2015 \text {. }\end{array}$ & $\begin{array}{l}\text { A segregação dos RSS é deficiente, pois apresentou mistura de até } \\
79,6 \% \text { de resíduos comuns (grupo D) acondicionados como } \\
\text { resíduos infectantes (grupo A) e } 15,5 \% \text { destes acondicionados como } \\
\text { resíduos comuns. A pesquisa evidenciou problemas na gestão dos }\end{array}$ \\
\hline Scielo & Macapá, Amapá, Brasil ${ }^{(19)}$ & & & $\begin{array}{l}\text { RSS especialmente pela falta de apropriação do PGRSS como } \\
\text { política ambiental da instituição. }\end{array}$ \\
\hline \multirow[t]{2}{*}{ Web of Science } & $\begin{array}{l}\text { Conhecimento e prática de } \\
\text { trabalhadores, profissionais e } \\
\text { gestores sobre os resíduos de } \\
\text { serviços de saúde }^{(20)}\end{array}$ & $\begin{array}{l}\text { Mendes WC, } \\
\text { Figueiredo MLF, } \\
\text { Salmito MA, } \\
\text { Araújo EC, } \\
\text { Araújo TME }\end{array}$ & $\begin{array}{l}\text { J.res.: fundam. } \\
\text { care. Online } 7(4) \text { : } \\
\text { 3216-3226, } \\
\text { out/dez } 2015 \text {. }\end{array}$ & $\begin{array}{l}\text { Verificou-se a deficiência da capacidade instalada, inadequação dos } \\
\text { carros para transporte dos RSS e equipamentos de proteção } \\
\text { individual (EPI's), dos recipientes (depósitos, caixas e sacos), não } \\
\text { implantação do PGRSS, além da precariedade da capacitação dos } \\
\text { envolvidos no manejo dos RSS. Evidenciou-se déficit no } \\
\text { conhecimento dos trabalhadores, profissionais e gestores acerca do } \\
\text { PGRSS, bem como práticas inadequadas o manejo dos } \\
\text { RSS. }\end{array}$ \\
\hline & $\begin{array}{l}\text { Desafio no Gerenciamento de } \\
\text { Resíduos em Serviços Públicos } \\
\text { de Saúde }\end{array}$ & $\begin{array}{l}\text { Rizzon F, Cristine } \\
\text { Nodari CH, Reis } \\
\text { ZC }\end{array}$ & $\begin{array}{l}\text { Revista de Gestão } \\
\text { em Sistemas de } \\
\text { Saúde- RGSS } \\
4(1): 40-54 \\
\text { Janeiro/Junho } \\
2015 .\end{array}$ & $\begin{array}{l}\text { Foram evidenciadas problemáticas relacionadas a todas as etapas do } \\
\text { processo de gerenciamento dos RSS e entre outros aspectos da } \\
\text { gestão (acondicionamento dos resíduos, coleta e transporte interno, } \\
\text { fluxo de coleta interna, quantificação dos RSS, armazenamento } \\
\text { interno e externo, área de higienização, coleta e transporte externo, } \\
\text { tratamento, disposição final, política de gestão ambiental e } \\
\text { capacitação e treinamento). }\end{array}$ \\
\hline Scielo & $\begin{array}{l}\text { Waste management in non- } \\
\text { hospital emergency units }\end{array}$ & Severino PM et al & $\begin{array}{l}\text { Rev. Latino-Am. } \\
\text { Enfermagem } 21(\mathrm{~s} \\
\text { pe): (1-8), jan-fev } \\
2013 \text {. }\end{array}$ & $\begin{array}{l}\text { O estudo revela a inexistência de política institucional de } \\
\text { gerenciamento de resíduos, evidenciada por falhas nas etapas } \\
\text { operacionais que envolvem problemas de gestão, estrutura } \\
\text { física, recursos materiais e humanos das unidades. }\end{array}$ \\
\hline
\end{tabular}

Quanto aos objetivos, segundo as tabelas II e III, a maioria dos artigos visavam de uma forma geral analisar a gestão dos resíduos sólidos nos estabelecimentos de prestação de serviços de saúde, com relação à existência e obediência do Plano de Gerenciamento de Resíduos de Serviços de Saúde (PGRSS), sobretudo nas etapas do gerenciamento dos RSS (segregação; acondicionamento; identificação; coleta e transporte interno; armazenamento interno, temporário e externo e destinação) conforme a legislação vigente, e/ou averiguar o conhecimento dos profissionais de saúde e acadêmicos com relação à temática. 


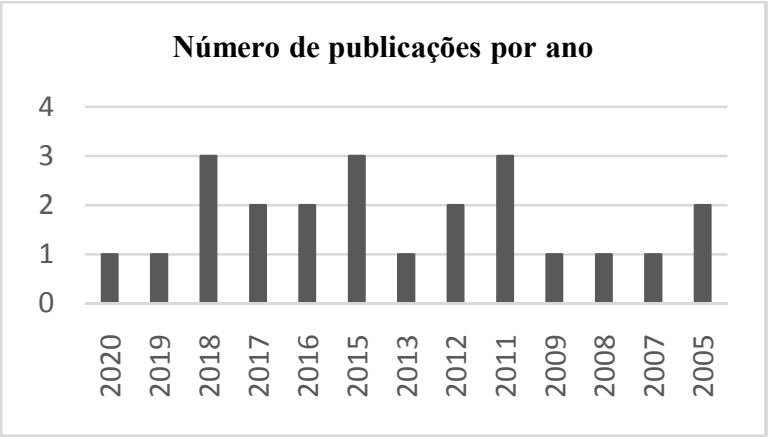

Gráfico 1. Número de publicações por artigos analisados.ano
RSS, inciso X, os geradores devem apresentar documento comprobatório da capacitação e treinamento dos funcionários envolvidos na prestação de serviço de limpeza e conservação que atuem no serviço, próprios ou terceiros de todas as unidades geradoras. Tal treinamento fornece aos funcionários um melhor conhecimento da legislação existente, contribuindo efetivamente no gerenciamento satisfatório. Em seu estudo, Ezirim e Agbo (2018), evidenciaram que, na Nigéria, ocorreu uma melhoria na segregação de resíduos, devido à implementação da política de resíduos de saúde, onde os profissionais de saúde foram treinados em gerenciamento de resíduos e foram desenvolvidos planos de gerenciamento de resíduos de estabelecimentos de saúde. Além disso, foram disponibilizadas ferramentas para a segregação, transporte e disposição de resíduos e identificados os funcionários responsáveis pelo gerenciamento.

Tabela III - Distribuição das pesquisas incluídas na revisão integrativa de acordo com procedência, título, nomes dos autores, periódico (vol, $\mathbf{n}^{\circ}$, pág, ano), considerações/temática, referentes aos anos de 2005-2012

\begin{tabular}{|c|c|c|c|c|}
\hline Procedência & Título & Autores & $\begin{array}{l}\text { Periódico (vol, } \mathrm{n}^{\circ}, \\
\text { pág, ano) }\end{array}$ & Considerações/temática \\
\hline Scielo & $\begin{array}{l}\text { Análise do sistema de gerenciamento } \\
\text { dos resíduos de serviços de saúde nos } \\
\text { municípios da bacia hidrográfica do } \\
\text { Rio dos Sinos, Rio Grande do Sul, } \\
\text { Brasil }^{(23)}\end{array}$ & $\begin{array}{l}\text { Gomes, LP, } \\
\text { Esteves, RVR }\end{array}$ & $\begin{array}{l}\text { EngSanitAmbient } \\
\text { 17(4): } \quad 377-384 \text {, } \\
\text { out/dez } 2012 \text {. }\end{array}$ & $\begin{array}{l}\text { Os resultados indicam que } 48,6 \% \text { dos estabelecimentos de } \\
\text { saúde atendem corretamente à legislação específica brasileira, } \\
\text { verificando ainda uma melhor gestão para os estabelecimentos } \\
\text { privados. }\end{array}$ \\
\hline Scielo & $\begin{array}{l}\text { Conhecimento de enfermeiros da } \\
\text { Estratégia Saúde da Família sobre } \\
\text { resíduos dos serviços de saúde }\end{array}$ & $\begin{array}{l}\text { Santos MA, } \\
\text { Souza, Oliveira A }\end{array}$ & $\begin{array}{l}\text { RevBrasEnferm } \\
65(4): \quad 645-52 \text {, jul- } \\
\text { ago } 2012 \text {. }\end{array}$ & $\begin{array}{l}\text { Apesar da existência de um significativo conhecimento sobre o } \\
\text { tema, ainda há necessidade de trabalhos de conscientização e } \\
\text { desenvolvimento de práticas adequadas de gerenciamento de } \\
\text { Resíduos de Serviços de Saúde. }\end{array}$ \\
\hline Scielo & $\begin{array}{l}\text { Modelo de implantação de plano de } \\
\text { gerenciamento de resíduos no } \\
\text { laboratório clínico }^{(25)}\end{array}$ & $\begin{array}{l}\text { Gonçalves EMN } \\
\text { et al }\end{array}$ & $\begin{array}{l}\text { J. Bras. Patol. Med. } \\
\text { Lab. [online] 47(3): } \\
\text { 249-255, 2011. }\end{array}$ & $\begin{array}{l}\text { O PGRSS facilitou a comunicação, a disseminação da } \\
\text { informação, a conscientização, a formação da competência e o } \\
\text { treinamento de todos os envolvidos. Várias atividades foram } \\
\text { desenvolvidas simultaneamente, resultando em mudanças } \\
\text { comportamentais. }\end{array}$ \\
\hline Scielo & $\begin{array}{l}\text { Vulnerabilidade no manejo dos } \\
\text { resíduos de serviços de saúde de João } \\
\text { Pessoa (PB, Brasil) }\end{array}$ & Ramos YS et al & $\begin{array}{l}\text { Ciênc. } \quad \text { saúde } \\
\text { coletiva }[\text { online] } \\
\text { 16(8): } \quad 3553-60, \\
\text { 2011. }\end{array}$ & $\begin{array}{l}\text { A vulnerabilidade do manejo dos RSS de João Pessoa - PB foi } \\
\text { significativo } 48,02 \% \text {, traduzindo a não observância dos } \\
\text { elementos legais e tático-operacionais do processo, podendo } \\
\text { contribuir para a deterioração da saúde ambiental e coletiva, } \\
\text { acarretando problema de saúde pública. }\end{array}$ \\
\hline Scielo & $\begin{array}{llll}\text { Resíduos sólidos } & \text { de serviços } & \text { de } \\
\text { saúde: uma } & \text { fotografia } & \text { do } \\
\text { comprometimento } & \text { da equipe } & \text { de } \\
\text { enfermagem }^{(27)} & & & \end{array}$ & $\begin{array}{l}\text { Doi KM, Moura } \\
\text { GMSS }\end{array}$ & $\begin{array}{l}\text { Rev. } \\
\text { Enferm. } \\
\text { 32(2): } \\
\text { 2011. }\end{array}$ & $\begin{array}{l}\text { Constatou-se que a abordagem referente aos resíduos sólidos de } \\
\text { serviços de saúde ainda é insuficiente, causando, assim, muitos } \\
\text { prejuízos no âmbito institucional e coletivo. }\end{array}$ \\
\hline Scielo & $\begin{array}{l}\text { Gerenciamento dos resíduos sólidos } \\
\text { dos serviços de saúde: aspectos do } \\
\text { manejo interno no município de } \\
\text { Marituba, Pará, Brasil }{ }^{(28)}\end{array}$ & Sales CCL et al & $\begin{array}{l}\text { Ciênc. saúde } \\
\text { coletiva [online] } \\
\text { 14(6): 2231-2238, } \\
2009 .\end{array}$ & $\begin{array}{l}\text { De modo geral, neste estudo, as normas federais não eram } \\
\text { atendidas e o geren-ciamento de RSSS dos estabelecimentos de } \\
\text { saúde necessita de adequação na realização de todas asetapas do } \\
\text { manejo, para controlar e diminuir os riscos e reduzir a } \\
\text { quantidade de resíduos. }\end{array}$ \\
\hline Scielo & $\begin{array}{l}\text { Proposta de um modelo de gestão } \\
\text { ambiental para os serviços de } \\
\text { Nefrologia }{ }^{(29)}\end{array}$ & $\begin{array}{l}\text { Burg G, Silveira } \\
\text { DD }\end{array}$ & $\begin{array}{l}\text { Acta paul. } \\
\text { enferm. [online] } \\
\text { 21(spe): 192-197, } \\
\text { 2008. }\end{array}$ & $\begin{array}{l}\text { A implantação do sistema proposto nos serviços trouxe } \\
\text { inúmeros benefícios que vão desde a redução de resíduos e } \\
\text { efluentes líquidos; preservação dos recursos naturais; uso de } \\
\text { materiais e insumos mais ambientalmente corretos; redução de } \\
\text { custos; preservação da saúde humana e do meio ambiente. }\end{array}$ \\
\hline Scielo & $\begin{array}{l}\text { O processo de formação em saúde: o } \\
\text { saber resíduos sólidos de serviços de } \\
\text { saúde em vivências práticas }{ }^{(30)}\end{array}$ & $\begin{array}{l}\text { Corrêa LB, } \\
\text { Lunardi VL, De } \\
\text { Conto SM }\end{array}$ & $\begin{array}{l}\text { RevBrasEnferm } \\
60(1): 21-5,2007 .\end{array}$ & $\begin{array}{l}\text { É possível afirmar } \text { que os } \\
\text { profissionais vivenciam ações relacionadas à segregação, } \\
\text { acondicionamento e coleta em alguns ambientes, porém sem } \\
\text { uma compreensão da totalidade deste manejo. }\end{array}$ \\
\hline Scielo & $\begin{array}{l}\text { Diagnóstico dos resíduos de serviços } \\
\text { de saúde no interior do Rio Grande do } \\
\text { Sul }{ }^{(31)}\end{array}$ & $\begin{array}{l}\text { Silva CE, Hoppe } \\
\text { AE }\end{array}$ & $\begin{array}{l}\text { Eng. Sanit. Ambient. } \\
\text { 10(2): } \quad 146-151 \text {, } \\
\text { Apr./June 2005. }\end{array}$ & $\begin{array}{l}\text { Todos os estabelecimentos de saúde apresentaram falhas nas } \\
\text { várias fases da gestão de seus resíduos, não atendendo os } \\
\text { princípios preconizados na Resolução CONAMA N. 283/2001. }\end{array}$ \\
\hline Scielo & $\begin{array}{l}\text { Gerenciamento de resíduos sólidos de } \\
\text { odontologia em postos de saúde da } \\
\text { rede municipal de Belo Horizonte, } \\
\text { Brasil }^{(32)}\end{array}$ & $\begin{array}{l}\text { Nazar MW, } \\
\text { Pordeus IA, } \\
\text { Werneck MAF }\end{array}$ & $\begin{array}{l}\text { Rev Panam Salud } \\
\text { Publica/Pan Am J } \\
\text { Public Health 17(4): } \\
\text { 237-242 } 2005\end{array}$ & $\begin{array}{l}\text { Nenhuma unidade possuía um plano de gerenciamento de } \\
\text { resíduos de serviços de saúde. Os únicos itens em conformidade } \\
\text { com a legislação em todas as unidades foram: as agulhas e o } \\
\text { mercúrio eram segregados; eram utilizados recipientes de } \\
\text { papelão resistente com simbologia adequada para } \\
\text { acondicionamento dos resíduos perfurocortantes; e a coleta e } \\
\text { transporte internos de resíduos eram feitos diariamente. }\end{array}$ \\
\hline
\end{tabular}

\section{DISCUSSÃO}

Kistet al (2018), mostrou em seu estudo que o seu PPGRS estava atualizado, de acordo com a legislação vigente em todas as etapas: segregação, acondicionamento, coleta, armazenamento, transporte, tratamento e disposição final; contudo o estudo também demonstrou a falta de uma política de treinamento que se enfatizasse a questão dos resíduos no estabelecimento estudado. De acordo com a RDC $\mathrm{n}^{\circ}$ $222 / 2018$, no seu artigo $6^{\circ}$, que trata dos deveres dos geradores dos
Logo, concluíram com estes achados que a política nacional de gerenciamento de resíduos de saúde neste país terá pouco ou nenhum impacto sobre o gerenciamento se todos os processos não forem implementados. Um dos exemplos mais reais e preocupantes da situação atual do gerenciamento de resíduos no Brasil, encontra-se no estudo de Teixeira et al (2018) no qual foi analisada a gestão dos RSS e o conhecimentos dos profissionais da enfermagem acerca da legislação vigente. Nos seus achados, $73,7 \%$ dos entrevistados afirmaram desconhecer a classificação dos resíduos e $36,8 \%$ desconheciam os cuidados específicos para cada tipo de resíduo. 
Fatos como esse podem aumentar os riscos de manuseio e segregação inadequado dos resíduos. Dentro dessa magnitude, Silva et al. (2016) apontaram em seu estudo que alguns profissionais da saúde, como exemplo os técnicos de enfermagem, além de estarem, frequentemente em contato com materiais infectantes, também apresentam uma maior constância de manipulação com os materiais perfurantes e cortantes em relação aos demais profissionais, o que evidencia mais uma vez a importância de um PPGRS com a implementação de todas as suas etapas, sobretudo com capacitações frequentes para estes e os demais profissionais. No tocante aos resultados e conclusões dos estudos, estes demonstraram que a maior parte dos estabelecimentos de saúde estudados não tinham uma gestão/gerenciamento dos RSS adequada, de acordo com a legislação vigente na época da realização do estudo, representando $86,9 \%$ dos estudos. Além disso, a maioria dos profissionais/acadêmicos também não possuíam conhecimento suficiente acerca da temática apresentada, nem tampouco puderam obter este conhecimento de acordo com a sua devida importância durante os anos de estudo na graduação, o que contribui diretamente para um gerenciamento de resíduos inadequado. Ademais, apenas um estudo abordou a legislação atual com a nova Resolução da Diretoria Colegiada (RDC) $\mathrm{n}^{\circ} 222$, de 28 de março de 2018 a qual regulamenta as boas práticas de gerenciamento dos RSS e dá outras providências, evidenciando que a produção científica nesta área necessita ser mais ativa e atualizada.

A presente revisão integrativa evidenciou por meio da literatura científica encontrada nas bases de dados escolhidas que, a gestão/gerenciamento dos resíduos dos serviços de saúde encontra-se prejudicada e necessita de ajustes na maioria dos estabelecimentos de saúde estudados, principalmente em relação à construção de Planos de Gerenciamento de Resíduos de Saúde, com a implementação de todas as suas etapas e ênfase nas capacitações dos profissionais que lidam diretamente com os RSS. Além disso, também foi apontado que há necessidade de olhar para a abordagem dos RSS nos cursos de graduação da área da saúde, para que os acadêmicos ingressem no mercado de trabalho devidamente habilitados em relação à temática e contribuam positivamente para uma boa gestão/gerenciamento nestes estabelecimentos. Foi possível perceber que a legislação foi atualizada recentemente com a RDC $222 / 2018$, porém as publicações anteriores a este período também demonstraram que a maior parte dos geradores de RSS ainda não haviam se adequado às normas vigentes da época, o que denota mais um desafio para a atualidade. O presente estudo apresentou limitações no que concerne ao número de publicações encontradas acerca da temática proposta, principalmente em relação a artigos atuais, o que torna a literatura deste tema ainda escassa. A gestão dos RSS é algo que deve ser trabalhado constantemente dentro dos estabelecimentos geradores destes resíduos e nos centros de estudo (escolas, universidades) devido à sua complexidade e importância para a saúde pública e ambiental. Logo, a conclusão dessa pesquisa contribuiu para realizar um diagnóstico apurado através das bases de dados consultadas acerca da gestão dos RSS tanto na teoria quanto na prática realizada nos estabelecimentos de saúde.Sendo assim, diante dos achados supracitados, ressalta-se a importância da discussão do tema em foco e sugere-se que novos estudos teóricos e de intervenção sejam realizados para criar subsídios a fim deauxiliar os geradores de RSS a se enquadrarem nas normas propostas pela legislação e dessa forma contribuir para uma gestão eficiente que vise a não geração, quando possível, e o reaproveitamento adequado destes resíduos.

Reconhecimentos: Andressa Pedroza Pereira trabalhou na concepção, delineamento, análise e interpretação dos dados e redação final e Maria de Fátima Martins, na metodologia e revisão crítica.

\section{REFERÊNCIAS}

Almeida FBD, Bilyk C, Sieben PG. Gestão de resíduos sólidos urbanos: impactos ambientais e o processo de inclusão social dos catadores de lixo. Gest.Tecnol.Inov. [periódico na Internet]. 2018; [acessado 2018 nov 20]; 2(1): 12-25. Disponível
em:Artigo2-n4-Gestão-de-resíduos-sólidos-urbanos.pdf (opet.com.br)

Amarante JAS, Rech TD, Siegloch AE. Avaliação do gerenciamento dos resíduos de medicamentos e demais resíduos de serviços de saúde na Região Serrana de Santa Catarina. Eng Sanit Ambient.[periódico na Internet]. 2017 [acessado 2020 jan 19]; 22(2): 317-326. Disponível em: https://www.scielo.br/pdf/esa/ v22n2/1809-4457-esa-s1413_41522016150080.pdf.

Assis MC, Gomes VP, Balista WC, Freitas RD.Use of performance indicators to assess the solid waste management of health services. In:Anais da Academia Brasileira de Ciências; 2017. p.2445-2460.

Associação Brasileira de Empresas de Limpeza Pública e Resíduos Especiais. Panorama dos resíduos sólidos no Brasil - 2017. [Internet]. 2017; [acessado 2020 jan 20]; Disponível em: http://abrelpe.org.br/panorama/2017.pdf.

Brasil. Agência Nacional de Vigilância Sanitária. Resolução da Diretoria Colegiada - RDC $\mathrm{n}^{\circ} 222$, de 28 de março de 2018.Regulamenta as Boas Práticas de Gerenciamento dos Resíduos de Serviços de Saúde e dá outras providências. Brasília: Anvisa 2018.

Burg G, Silveira DD. Proposta de um modelo de gestão ambiental para os serviços de nefrologia.Acta Paul Enferm. [periódico na Internet]. 2008 [acessado 2020 jan 13]; 21(Número Especial). Disponível em: https://www.scielo.br/pdf/ape/v21nspe/ a10v21ns.pdf.

Conselho Nacional do Meio Ambiente (CONAMA). Resolução ${ }^{\circ}$ 358 , de 29 de abril de 2005. Dispõe sobre o tratamento e a disposição final dos resíduos dos serviços de saúde e dá outras providências. Diário Oficial da República Federativa do Brasil 2005; 04 maio. Seção I, p. 63 - 65.

Corrêa LB, Lunardi VL, De Conto SM. O processo de formação em saúde: o saber resíduos sólidos de serviços de saúde em vivências práticas.RevBrasEnferm. [periódico na Internet]. 2007 [acessado 2020 jan 14];30(1):21-5. Disponível em: https://www.scielo.br/pdf/reben/v60n1/a04v60n1.pdf.

DelevatiDS, Castro MMRS, Ries EF, Bayer VML, Rocha VMP. Desafios na gestão de resíduos de estabelecimentos de saúde públicos perante a RDC 222/18.Saúde Debate. [periódico na Internet]. 2019 [acessado 2020 jan 20]; 43 (no.esp.): 190-199. Disponível em: https://www.scielo.br/pdf/sdeb/v43nspe3/01031104-sdeb-43-spe03-0190.pdf.

Doi KM, Moura GMSS. Resíduos sólidos de serviços de saúde:uma fotografia do comprometimento da equipe de enfermagem. Rev. GaúchaEnferm. [periódicona Internet]. 2011 [acessado 202015 jan]; 32(2): 338-344.Disponívelem: https://www.scielo.br/pdf/ rgenf/v32n2/a18v32n2.pdf.

Ezirim I, Agbo F. Role of national policy in improving health care waste management in Nigeria. $J$ Health Pollut. [periódico na Internet]. 2018 [acessado 2020 jan 16]; 8(19):1-8. Disponível em: https://www.ncbi.nlm.nih.gov/pmc/articles/PMC6257174/.

Gomes LP, Esteves RVR.Análise do sistema de gerenciamento dos resíduos de serviços de saúde nos municípios da bacia hidrográfica do Rio dos Sinos, Rio Grande do Sul, Brasil. Eng.Sanit. Ambient. [periódicona Internet]. 2012[acessado 2020 jan 15]; 17(4):377-384. Disponível em: https://www.scielo.br $/ \mathrm{pdf} / \mathrm{esa} / \mathrm{v} 17 \mathrm{n} 4 / \mathrm{v} 17 \mathrm{n} 4 \mathrm{a} 04$.

Gonçalves EMN, Santos CBS, Badaró MLS, Farias VA, Rodrigues E, Mendes ME,SumitaNM.Modelo de implantação de plano de gerenciamento de resíduos no laboratório clínico. $J$ BrasPatolMed Lab. [periódico na Internet]. 2011 [acessado 2020 jan 14]; 47(3): 249-255. Disponível em: https://www.scielo.br/ $\mathrm{pdf} / \mathrm{jbpml} / \mathrm{v} 47 \mathrm{n} 3 / \mathrm{v} 47 \mathrm{n} 3 \mathrm{a} 08 . p d f$.

Kist LT, Rosa FR, Moraes JAR,Machado EL.Diagnosisof hospital waste management in Vale do Rio Pardo, Rio Grande do Sul, Brazil. Rev. Gest. Ambient. Sustentabilidade. [periódico na Internet]. 2018 [acessado 2020 jan 21]; 7(3): 554-569. Disponível em: https://www.redalyc.org/jatsRepo/4716/ 471659747012/471659747012.pdf.

Maders GR, Cunha HFA. Análise da gestão e gerenciamento dos resíduos de serviços de saúde (RSS) do Hospital de Emergência de Macapá, Amapá, Brasil.EngSanitAmbient. [periódico na 
Internet]. 2015 [acessado 2020 jan 19]; 20(3): 379-388. Disponível em: https://www.scielo.br/pdf/esa/v20n3/1413-4152esa-20-03-00379.pdf.

Melo SB, Zajac MAL, Régis MM, Oliveira JES. Recuperação de papéis recicláveis: Aspectos da reciclagem e do reaproveitamento em ambiente hospitalar.In: Anais do VI SINGEP-São Paulo - SP - Brasil; 2017; São Paulo. p. 1-16.

Mendes KDS, Silveira RCCP, Galvão CM. Revisão integrativa: método de pesquisa para a incorporação de evidências na saúde e na enfermagem.Texto \&amp; Contexto Enfermagem. [período na Internet]. 2008 [acessado 2020 jan 19]; 17(4): 758-764. Disponível em: https://www.scielo.br/pdf/tce/v17n4/18.pdf.

Mendes WC, Figueiredo MLF, Salmito MA, Araújo EC, Araújo TME.Conhecimento e prática de trabalhadores, profissionais e gestores sobre os resíduos de serviços de saúde. J. res.: fundam. care. online. [periódico na Internet]. 2015 [acessado 2020 jan 16]; 7(4): 3216-3226. Disponível em: https://dialnet.unirioja.es/ servlet/articulo?codigo $=5204807$.

Ministério da Saúde. Manual de gerenciamento de resíduos dos serviços de saúde. Brasília: Ministério da Saúde. [Internet] 2006; 182p. [acessado 2020 jan 20]; Disponível em:http://bvsms.saude.gov.br/bvs/publicacoes/manual_gerencia mento residuos.pdf.

Moreira $A \bar{M}$, Gunther WM. Solid waste management in primary healthcare centers: application of a facilitation tool.RevLatAm Enfermagem [periódico na Internet]. 2016 [acessado 2020 jan 19]; 24(e2768): 1-9. Disponível em: https://www.scielo.br/pdf/ rlae/v24/pt 0104-1169-rlae-24-02768.pdf.

Nazar MW, Pordeus IA, Werneck MAF. Gerenciamento de resíduos sólidos de odontologia em postos de saúde da rede municipal de Belo Horizonte.Rev Panam Salud. Publica. [periódico na Internet]. 2005 [acessado 202014 jan];17(4):237-42. Disponível em: https://www.scielosp.org/article/rpsp/2005.v17n4/237-242/.

Neves BC, Lima EPP. Condições da prestação dos serviços ambientais de coleta e destinação de resíduos de serviços de saúde em unidades básicas de saúde na cidade de Pelotas, RS, Brasil.EngSanitAmbient. [periódico na Internet]. 2019 [acessado 2020 jan 17]; 24(1): 61-69. Disponível em: https://www.scielo. br/pdf/esa/v24n1/1809-4457-esa-s1413-41522019172729.pdf.

Pereira MS, Alves SB, Souza ACS, Tipple AFV, Rezende FR, Rodrigues EG. Waste management in non-hospital emergency units. Rev Latino-Am Enfermagem. [periódiconaInternet]. 2013 [acessado 2020 jan 15];21(especial):259-66. Disponível em: https://www.scielo.br/scielo.php?script=sci_arttext\&pid=S010411692013000700032 .
Ramos YS, Pessoa YSRQ, Ramos YS, Netto FBA, Pessoa CEQ. Vulnerabilidade no manejo dos resíduos de serviços de saúde de João Pessoas (PB, Brasil).Ciência \& Saúde Coletiva. [periódico na Internet]. 2011 [acessado 2020 jan 14];16(8):355360. Disponível em: https://www.scielo.br/pdf/csc/ v16n8/a23v16n8.pdf.

Rizzon F, NodariCH, Dos Reis ZC. Desafio no gerenciamento de resíduos em serviços públicos de saúde.Revista de Gestão em Sistemas de Saúde.[periódico na Internet]. 2015 [acessado 2020 jan 16]; 4(1): 40-54. Disponível em: http://www.revistargss. org.br/ojs/index.php/rgss/article/view/141/152.

Sales CCL, Spolti G, Lopes MSB, Lopes DF. Gerenciamento dos resíduos sólidos dos serviços de saúde: aspectos do manejo interno no município de Marituba, Pará, Brasil. Ciência \& Saúde Coletiva [periódico na Internet]. 2009 [acessado 202016 jan]; 14(6): 2231-2238. Disponível em: https://www.scielo.br/pdf/csc/ v14n6/32.pdf.

Santos MA, Souza AO. Conhecimento de enfermeiros da Estratégia Saúde da Família sobre resíduos dos serviços de saúde.RevBrasEnferm. [periódicona Internet]. 2012 [acessado 2020 jan 15]; 65(4): 645 - 652. Disponível em: https://www.scielo.br/pdf/reben/v65n4/a14v65n4.pdf.

Silva CE, Hoppe AE. Diagnósticos dos resíduos de serviços de saúde no interior do Rio Grande do Sul.Eng. Sanit. Ambient.[periódico na Internet]. 2005 [acessado 2020 jan 13];10(20): 46-51. Disponível em: https://www.scielo.br/pdf/esa/v10n2/ a08v10n2.pdf.

Silva DF, Sperling EV, Barros RTV. Avaliação do gerenciamento dos resíduos de serviços de saúde em municípios da região metropolitana de Belo Horizonte (Brasil).EngSanitAmbient. [periódico na Internet].2014 [acessado 2018 nov 23]; 19(3): 251262. Disponível em: https://www.scielo.br/pdf/esa/v19n3/14134152-esa-19-03-00251.pdf.

Souza MT, Silva MD, Carvalho R. Revisão integrativa: o que é e como fazer.Einstein. [período na Internet]. 2010 [acessado 2020 jan 17]; 8(1): 102-106.Disponívelem: https://www.scielo.br/ pdf/eins/v8n1/pt_1679-4508-eins-8-1-0102.pdf.

Teixeira MV, Echevarría-Guanilo ME, Knuth FG, Ceolin T. Assessment of The Waste Management in Basic Health Units From a South Brazilian City. J. res.: fundam. care. online. [periódicona Internet].2018 [acessado 2020 jan 20]; 10(3):82483. Disponível em: http://www.seer.unirio.br/index.php/ cuidadofundamental/article/view/6237/pdf.

UrsiES.Prevenção de lesões de pele no perioperatório: revisão integrativa da literatura. [dissertação]. Ribeirão Preto: Universidade de São Paulo; 2005. 